\title{
Structural and Optoelectronic Properties of In-Zn-S sprayed Layers
}

\author{
S. LAzzez ${ }^{a}$, K. BoubakeR ${ }^{a, *}$, T. Ben NAsrallah ${ }^{a}, \mathrm{M} \mathrm{MnARI}^{b}$, \\ R. Chtourou $^{c}$, M. Amlouk ${ }^{a}$ And S. Belgacem ${ }^{a}$ \\ ${ }^{a}$ Unité de Physique des Dispositifs á Semiconducteurs \\ - Faculté des Sciences de Tunis \\ Université de Tunis El Manar, 2092 Tunis, Tunisia \\ ${ }^{b}$ Laboratoire de Chimie Analytique et d'Electrochimie \\ - Faculté des Sciences de Tunis \\ Université de Tunis El Manar, 2092 Tunis, Tunisia \\ ${ }^{c}$ Laboratoire de Photovoltaïque et des Matériaux Semiconducteurs \\ Institut National de Recherche Scientifique et Technique (INRST) \\ Hammam-Lif, Tunisia \\ (Received May 14, 2008)
}

In this work, In-Zn-S thin layers were prepared using the spray pyrolysis technique on glass substrates at $320^{\circ} \mathrm{C}$. The molar ratio between zinc and indium $x=\left[\mathrm{Zn}^{2+}\right] /\left[\mathrm{In}^{3+}\right]$ was varied in $0-0.4$ domain whereas $\left[\mathrm{S}^{2-}\right] /\left[\mathrm{In}^{3+}\right]$ one was taken constant equal to 2 . The atomic composition was carried out with the atomic absorption. The structural study of all layers via $\mathrm{X}$-ray diffraction and atomic force microscopy shows that the layer, obtained using $x=0$ is formed by binary material $\operatorname{In}_{2} \mathrm{~S}_{3}$ with a principal orientation along (400). When the composition increases the same study depicts the presence of other materials such as $\mathrm{ZnO}, \mathrm{ZnS}$, and $\mathrm{ZnIn}_{2} \mathrm{~S}_{4}$. On the contrary, for $x=0.4$, the film is mainly formed by the ternary compound $\mathrm{ZnIn}_{2} \mathrm{~S}_{4}$ which crystallizes in cubic phase. Moreover, the optical analysis via the transmittance, reflectance as well as the photocurrent reveals that the band gap energy $E_{\mathrm{g}}$ increases slightly as a function of the $x$ composition $\left(E_{\mathrm{g}}\right.$ varies from 2.6 to $2.9 \mathrm{eV}$ ).

PACS numbers: 79.60.Dp, 78.66.Li, 78.30.Am

\section{Introduction}

In-Zn-S alloys, which are made out of the binary $\operatorname{In}_{2} \mathrm{~S}_{3}$ compounds, are elements of high performance in the setup of the solar cells. In fact, the addi-

*corresponding author; e-mail: boubaker_karem@yahoo.com 
tion of zinc in the binary relatively weak-gapped $\operatorname{In}_{2} \mathrm{~S}_{3}(2-2.6)$ would be expectedly able to widen the band gap energy of the material and therefore improve the $\mathrm{In}_{2} \mathrm{~S}_{3} / \mathrm{CuInS}_{2}$-type solar cells photovoltaic output. Such alloys are prepared entirely by chemical pulverization in liquid phase (spray) and their energetic conversion ratio is enough promising (9.5\%) [1]. In the present work we studied structural, morphological, optical, and photoconductive properties as well as the chemical composition of semiconductor compounded zinc-indium sulphide layers regarding their uses as optical windows in the photovoltaic devices, and in order to avoid the use of the cadmium sulphide which is used less and less due to cadmium element toxicity.

In the same way, we took into account the presence of zinc-related secondary phases $(\mathrm{ZnS}$ and $\mathrm{ZnO})$ while evaluating photoconductive properties of the elaborated $\mathrm{In}-\mathrm{Zn}-\mathrm{S}$ layers.

\section{Experimental details}

\subsection{Layers elaboration}

The In-Zn-S thin layers have been prepared by the technique of reactive chemical pulverization in liquid phase (Spray) under a substrate temperature $T_{\mathrm{s}}=$ $320^{\circ} \mathrm{C}$. We used the following precursors: indium chloride $\mathrm{InCl}_{3}\left(\left[\mathrm{In}^{3+}\right]=10^{-2} \mathrm{M}\right)$ and thiourea $\left(\left[\mathrm{S}^{2-}\right]=2 \times 10^{-2} \mathrm{M}\right)$. The zinc chloride $\mathrm{ZnCl}_{2}$, as a unique zinc supply, was fixed through the variable ratio concentration $x=\left[Z_{n}^{2+}\right] /\left[I_{n}^{3+}\right]$ inside the starting solution. Values of $x$ were $0.1,0.2,0.3$ and 0.4 . The vector gas (nitrogen) and solution respective flow rates were $4 \mathrm{~L} \mathrm{~min}^{-1}$ and $2 \mathrm{~mL} \mathrm{~min}{ }^{-1}$. The used substrate was a massive $20 \times 20 \times 1 \mathrm{~mm}^{3}$ glass substrates. Performed layers mean thickness under these conditions was of the order of $1 \mu \mathrm{m}$.

\subsection{Characterization techniques}

The X-ray diffraction analysis of the prepared layers were performed by a copper-source diffractometer (Panalytical X Pert PROMPD), with the wavelength $(\lambda=1.54056 \AA)$.

The optical transmission and reflection measures has been achieved by means of a spectrophotometer (Shimadzu UV 3100S) dotted with an integrating sphere (LISR 3200). The spectrophotometer consists of double-beam monochromator with enough energy to make several types of accurate measures in a wide wavelength range (220-1800 $\mathrm{nm})$.

The morphological investigation has been achieved by atomic force microscopy (AFM) using a VEECO digital instrument 3A apparatus, operating in contact mode. The atomic absorption was measured by a Perkin ELMER 3100type device.

Concerning photoconductivity measurements, the light excitation was issued from a $0.25 \mathrm{~m}$ Jobin Yvon monochromator including a 600 lines $/ \mathrm{mm}$ diffractor illuminated by an Iode-Quartz $250 \mathrm{~W}$ bulk. The photocurrent generated by the studied layer, is measured using a Stanford-type numeric synchronous detection 
linked to the optical excitation by a mechanical light chopper. These measurements are done under room temperature in the wavelength range: $300-800 \mathrm{~nm}$. Several modulation frequencies $f(5-70 \mathrm{~Hz})$ and sample polarization voltages $U(1-10 \mathrm{~V})$ have been tested. Ohmic contacts were achieved by vacuum indium deposit on the surface of the thin layer. The distance inter-electrodes was $l=4 \mathrm{~mm}$.

\section{Results and discussion}

3.1. X-ray diffraction analysis and layers composition

Figure 1 shows the zinc-free $(x=0)$ binary In-S diffraction diagram. One can notice the appearance of the orientations (311), (400) and (511), which are characteristic of the $\beta$ - $\operatorname{In}_{2} \mathrm{~S}_{3}$ material. The crystallites privileged orientation is (400). This result is similar to the earlier found ones [2].

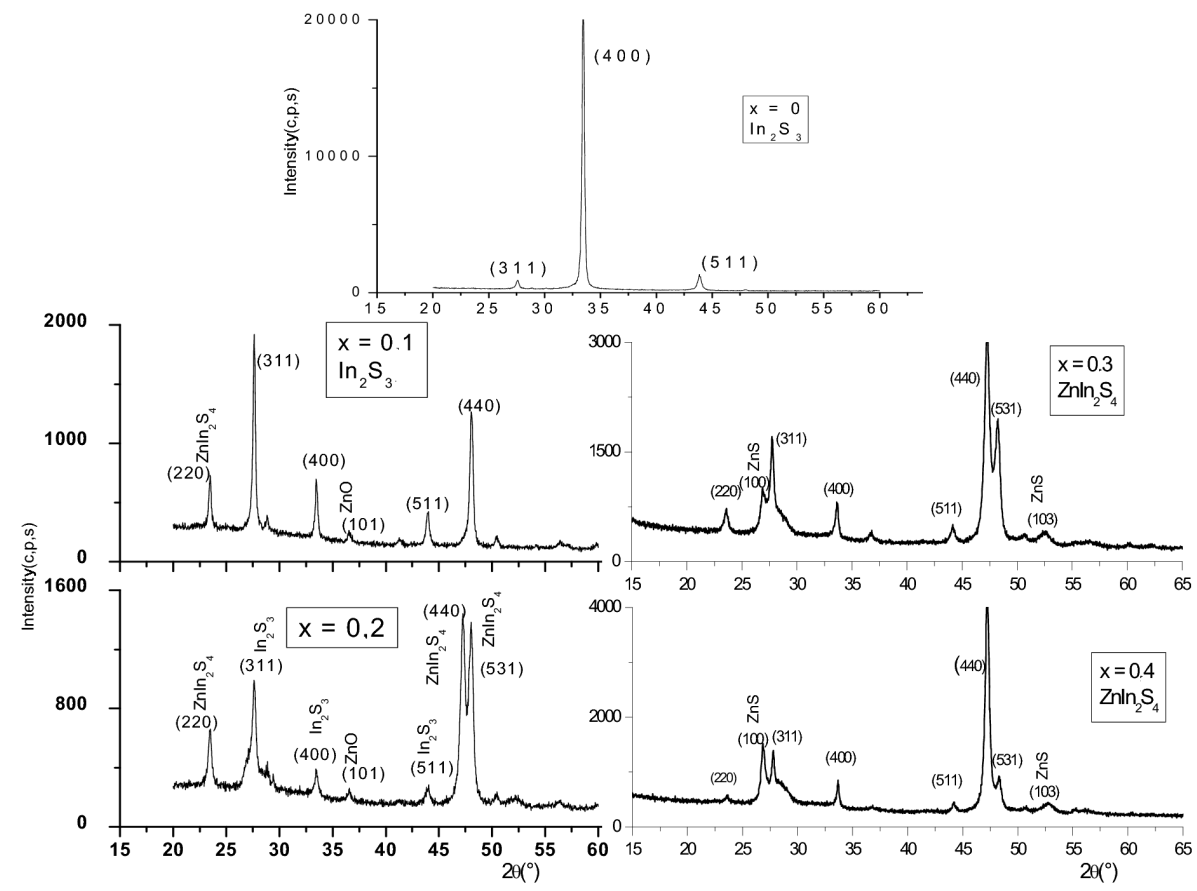

Fig. 1. X-ray diffraction spectra of In-Zn-S thin films prepared at different $x$ composition.

In the same figure (Fig. 1) we represented the In-Zn-S alloys diffractograms versus the $x$ composition. We could note that the layer, prepared using a composition $x=0.1$ is a mixture of binary $\mathrm{In}_{2} \mathrm{~S}_{3}, \mathrm{ZnO}$, and the ternary $\mathrm{ZnIn}_{2} \mathrm{~S}_{4}$. When $x$ increases, the corresponding diffractograms show the apparition of the binary $\mathrm{ZnS}$ starting from $x=0.3$. Particularly for $x=0.4$, the ternary $\mathrm{ZnIn}_{2} \mathrm{~S}_{4}$ appear in the same way, more and more in favor of binary phases binaires $\operatorname{In}_{2} \mathrm{~S}_{3}$ and $\mathrm{ZnS}$. In fact, for this composition, the layer of indium sulphide and zinc, prepared by this 
technique of spray, crystallize according to the privileged direction (440) of the cubic variety $(a=10.62 \AA$ ) (JCPDS 00-048-1778), Fig. 1 and Table I. The same ternary compound, synthesized earlier by chemical transport methods, crystallized under the hexagonal variety $(a=3.88 \AA$ and $c=37.02 \AA)[3,4]$. The present work has for merit the fact of giving, for the first time, the possibility of synthesis of this ternary $\mathrm{ZnIn}_{2} \mathrm{~S}_{4}$ from the spray pyrolysis method.

TABLE I

Relative intensity of X-ray diffraction peaks of $\mathrm{ZnIn}_{2} \mathrm{~S}_{4}$.

\begin{tabular}{c|c|c}
\hline \hline Orientation degree & JCPDS & $\begin{array}{c}\mathrm{ZnIn}_{2} \mathrm{~S}_{4} \text { sprayed layer } \\
(x=0.4)\end{array}$ \\
\hline$I_{311} / I_{440}$ & 1.72 & 0.24 \\
$I_{400} / I_{440}$ & 0.72 & 0.11 \\
$I_{531} / I_{440}$ & 0.10 & 0.15
\end{tabular}

The In- $\mathrm{Zn}-\mathrm{S}$ thin layers atomic absorption study results, gathered in Fig. 2, show a quasi-linear dependence between the ratio $x_{\mathrm{s}}=\left[Z_{n}^{2+}\right] /\left[\left[_{n}^{3+}\right]\right.$ in the layer solid phase, and the ratio $x=\left[Z_{n}^{2+}\right] /\left[I_{n}^{3+}\right]$ inside the starting solution. The value

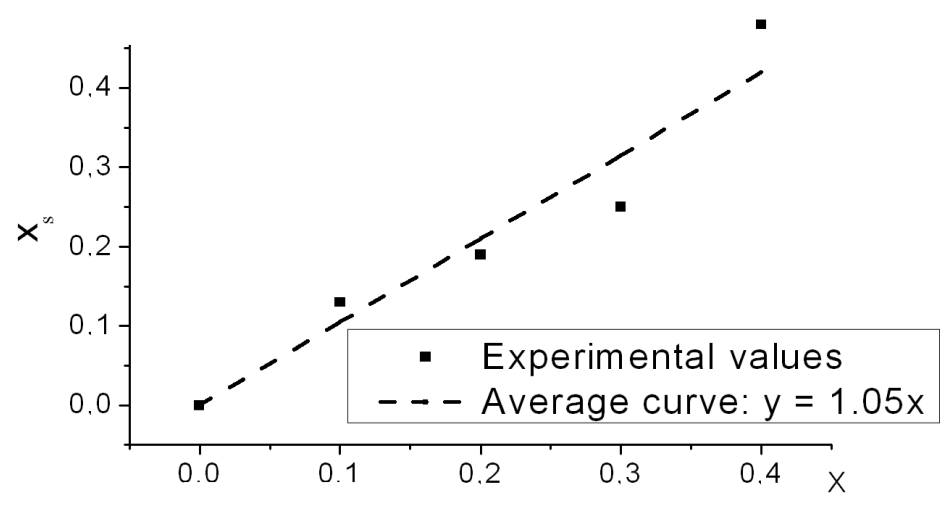

Fig. 2. Variation of $\left[\mathrm{Zn}^{2+}\right] /\left[\mathrm{In}^{3+}\right]_{\text {solid }}$ as a function of the $x$ composition in solution, determined by the atomic absorption technique.

$x_{\mathrm{s}}$, in solid phase is close to 0.48 (near to 0.5 ) for $x=0.4$ in liquid phase. This result is in good agreement with those found by X-ray diffraction analysis, that revealed the presence of the ternary material $\mathrm{ZnIn}_{2} \mathrm{~S}_{4}$ for the same composition.

\subsection{Surface morphology}

The obtained layers have been analyzed by the atomic force microscope. Figure 3 regroups 2D and 3D micrographs of In-Zn-S thin layers, it shows that these layers are formed by crystallites separated by depressions that can be interpreted 
as crystallized zones separated by grain joints. From this feature, we can deduce that layers are inhomogeneous both in surface and in depth In the same way, Fig. 3 also shows that the shape of crystallites depends on $x$. In fact, for low values of
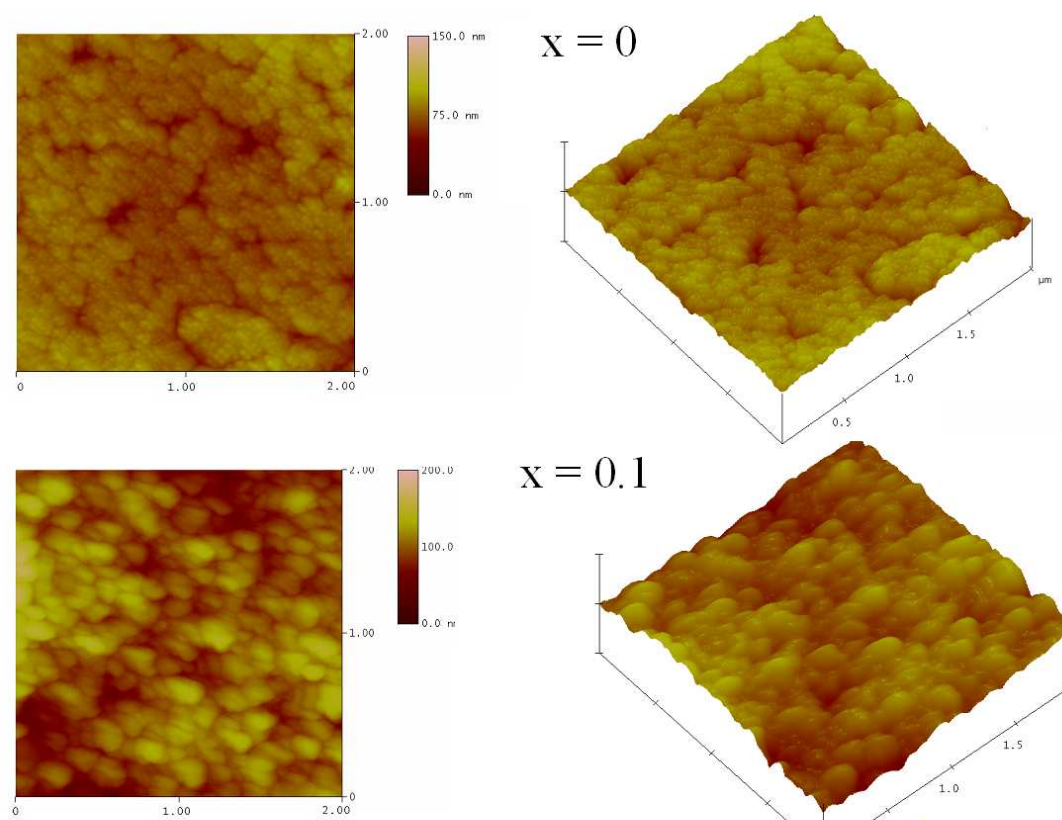

$\mathrm{x}=0.1$
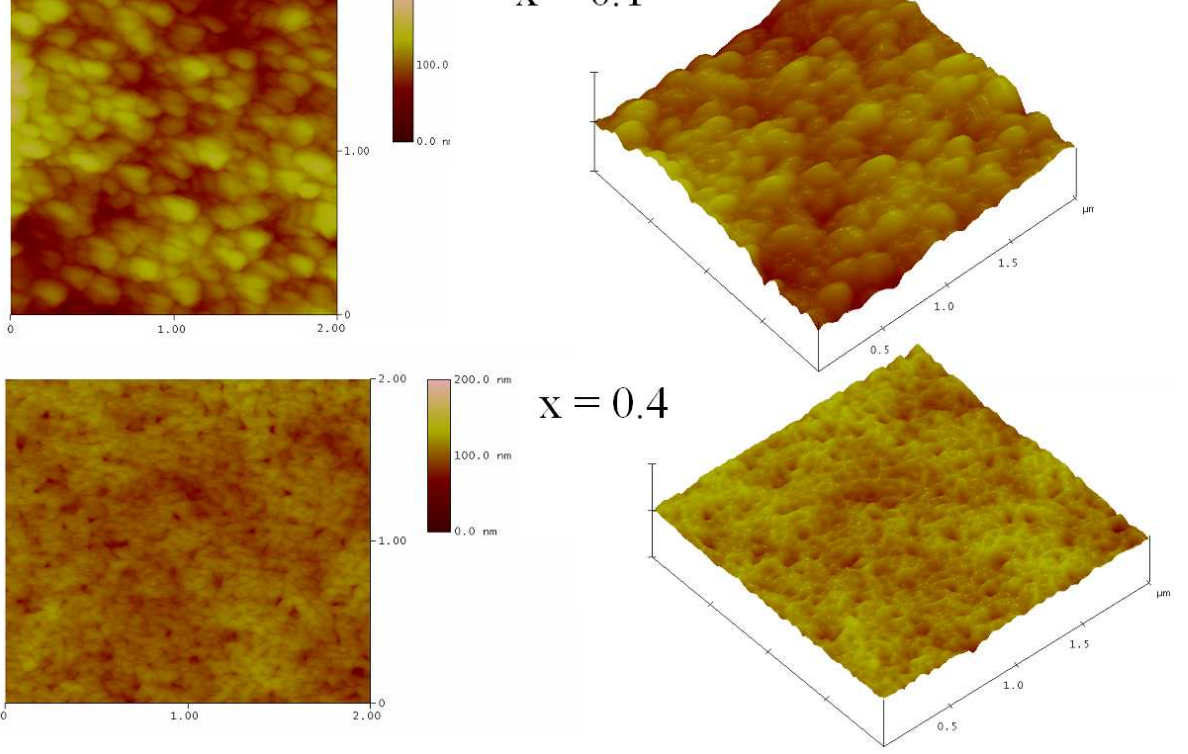

Fig. 3. 2D and 3D AFM micrographs of sprayed In-Zn-S [for $x=0,0.1$, and $x=0.4$.

TABLE II

Average of roughness and grain size values of the different In-Zn-S thin films with the zinc content $x$.

\begin{tabular}{c|c|c|c|c|c}
\hline \hline$x$ & 0 & 0.1 & 0.2 & 0.3 & 0.4 \\
\hline$R$ - average roughness [nm] & 8 & 19.7 & 11.5 & 14.4 & 8.7 \\
average diameter of grains [nm] & 30 & 72 & 30 & 36 & 42
\end{tabular}


$x(x \leq 0.2)$ heaps have a rounded shape. Oppositely, for higher values $(x \geq 0.3)$ the zinc gives a different aspect of the surface topography by yielding small fibers. These fibers seem to occur by fragmentation of the heaps. Otherwise, excepted the In-Zn-S layer prepared for $x=0.1$, when $x$ increases we note a reduction of the roughness and the size of crystallites. Table II gathers the size of grains and the mean roughness measurements done on a $2 \times 2 \mu \mathrm{m}^{2}$ surface of each sample.

\subsection{Optical properties}

Figures $4 \mathrm{a}$ and $\mathrm{b}$ represent the optical reflection and transmission spectra of the different alloys (In-Zn-S) deposited on glass. All these deposits are transparent enough in the visible (from 40 to $60 \%$ ) and in the infrared (70\%) domains. One also notices that the transparency is important for the sample characterized by $x=0.3$. In the interval of studied wavelengths the reflection has a mean value of $20 \%$.
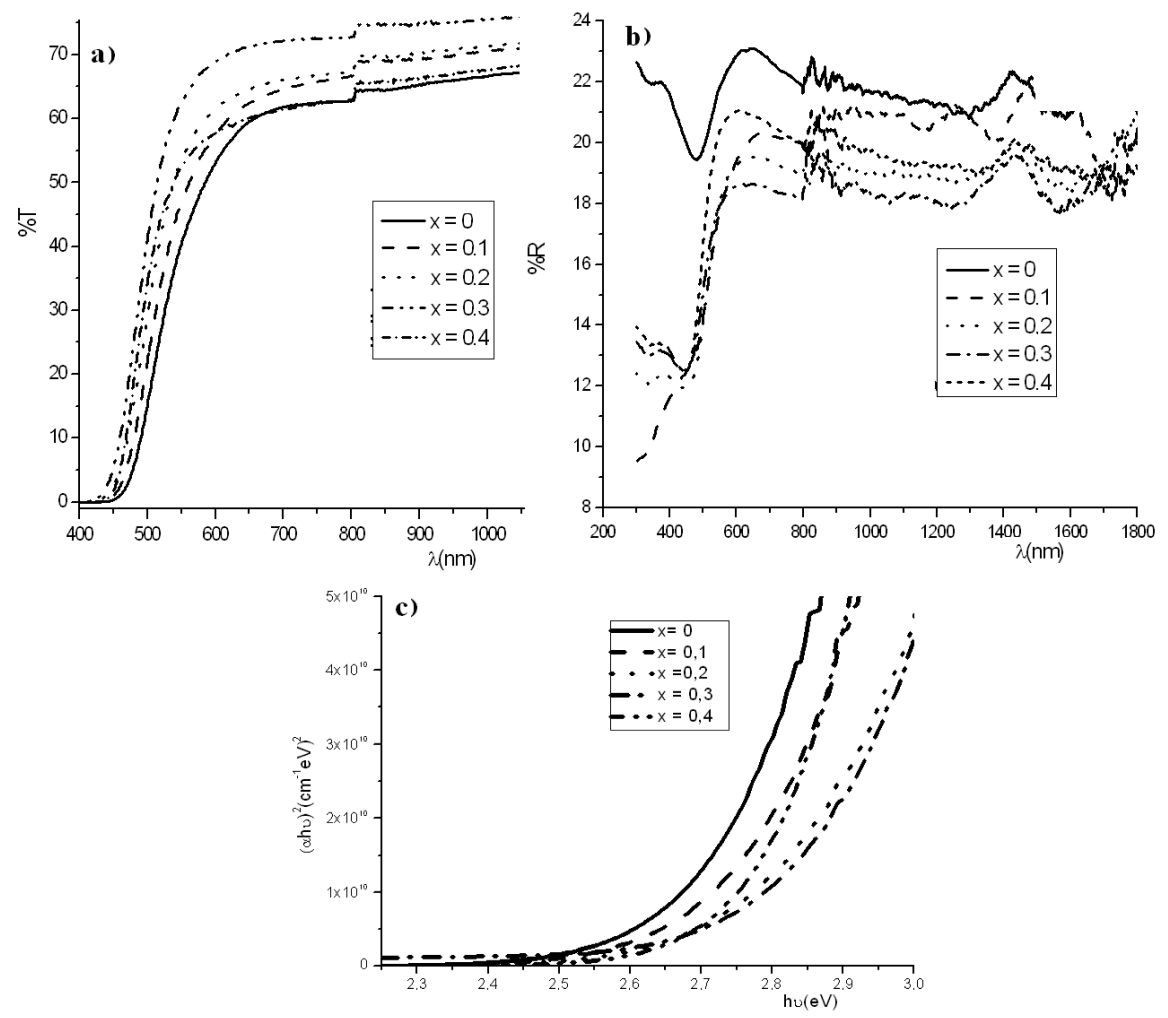

Fig. 4. (a) Optical transmittance of $\mathrm{In}-\mathrm{Zn}-\mathrm{S}$ thin films prepared at different ratio. (b) Optical reflectance of In-Zn-S thin films prepared at different ratio. (c) Variation of $(a h \nu)^{2}$ as a function of the energy $h \nu$ for different ratio $x$. 
We presented in the mean absorption zone the variation of $(\alpha h \nu)^{2}$ versus $(h \nu)$ for the incidental photons, and for the different layers, Fig. 4c. The absorption coefficient $\alpha$ has been determined in this zone from the approximated expression of the transmission

$$
T \approx(1-R)^{2} \mathrm{e}^{-\alpha d}
$$

The intersection of the tangent to this curve with the $x$-axis gives the value of the optical gap energy $E_{\mathrm{g}}$ in accordance with the following relation [5]:

$$
(\alpha h \nu)^{2} \approx A\left(h \nu-E_{\mathrm{g}}\right) ; \quad A=\text { const. }
$$

This relation is valid in the case of the direct transitions. The set of results concerning the variation of the energy of the band gap versus the composition $x$ are reported in Table III.

TABLE III

Band gap energy of In-Zn-S thin films with the zinc content.

\begin{tabular}{c|c|c|c|c|c}
\hline \hline$x$ & 0 & 0.1 & 0.2 & 0.3 & 0.4 \\
\hline$E_{\mathrm{g}}[\mathrm{eV}]$ & 2.65 & 2.76 & 2.83 & 2.90 & 2.80
\end{tabular}

One notices according to Table III that for $x \leq 0.3, E_{\mathrm{g}}$ increases with $x$, this is probably due to the presence of the binary compounds $\mathrm{ZnO}$ and $\mathrm{ZnS}$ that have relatively high optical gap energies $(\geq 3.2 \mathrm{eV})$. With regard to the synthesized layer using the $x=0.4$, the value of the forbidden band is $2.8 \mathrm{eV}$. This value is in the same range with the one found by Serpi [6] in a photoconductivity study of thin layers of the same ternary compound.

In the same line, an interesting feature of this ternary arises from the fact that there are two possible coordination sites from the indium atom, giving optical and electrical properties different from those of the related II-III-IV ternary semiconductors. Moreover, there is great interest in this material for possible nonlinear optical application [7-9].

\subsection{Photoconductivity measurement}

We present in Fig. 5 the $\mathrm{Zn}-\mathrm{In}-\mathrm{S}(0-0.4)$ thin layers photocurrent spectra versus the energy $h \nu$ of the incidental beam for different values of polarization voltage and different values of chopper frequencies. We note that for all samples the response increases with the polarization voltage under a constant frequency, while this response decreases with the increase in the frequency under fixed polarization.

The exploitation of the applied measures of enclosed $I_{\mathrm{ph}}$ in the mean absorption zone permits to estimate the value of the width of the forbidden band of the studied films. In fact, while standing in the mean absorption zone where $\alpha e \ll 1$, this photocurrent becomes appreciably proportional to $\alpha$, in accordance with the approximating relation $[10,11]$ : 

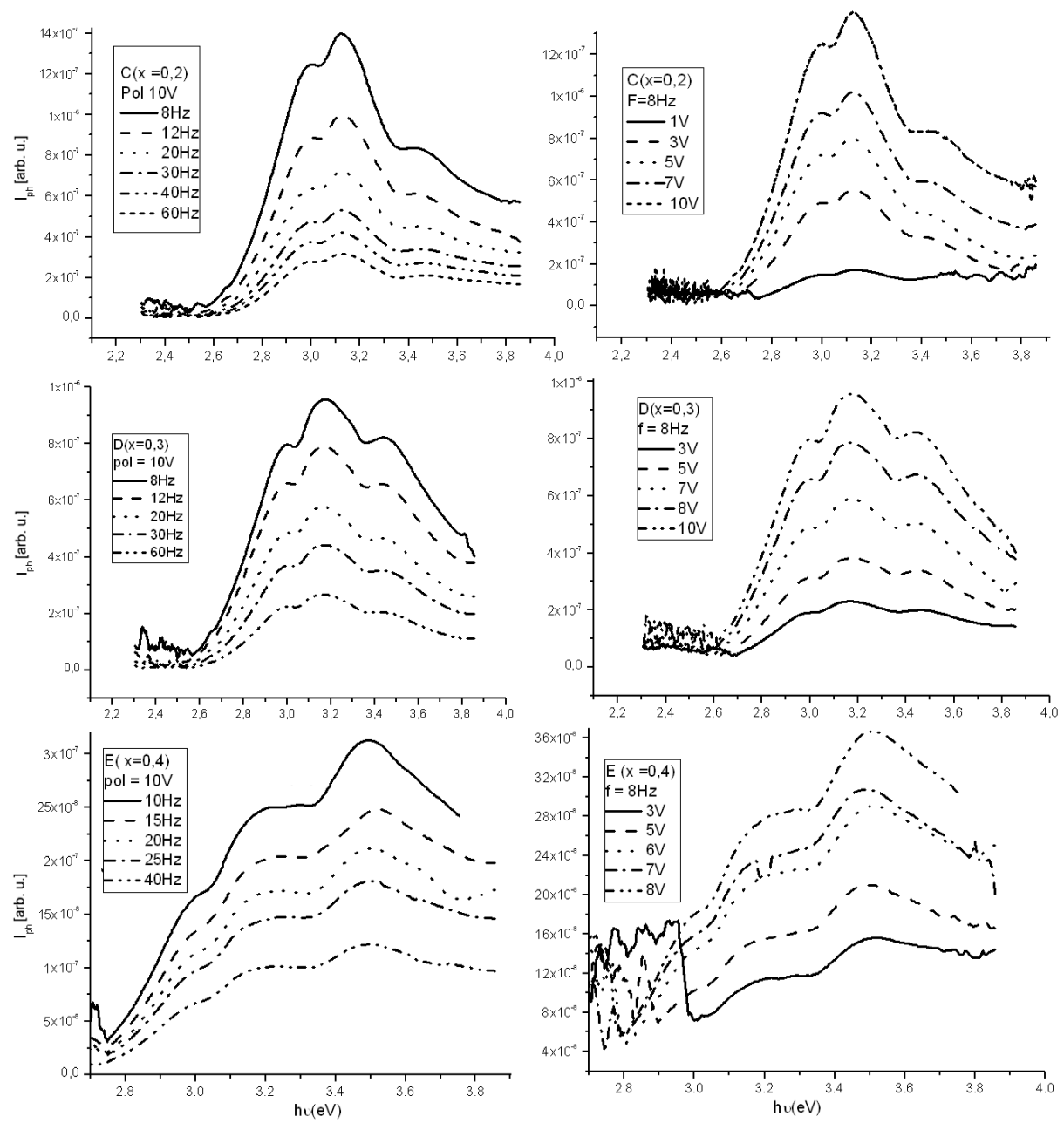

Fig. 5. Variation of the photocurrent $I_{\mathrm{ph}}$ as a function of the incident light energy at different bias voltage and different frequency modulation.

$$
I_{\mathrm{ph}} \cong \frac{\alpha e}{1+\frac{2 r}{w}}, \quad \text { with } \quad w=\frac{e}{\sqrt{D_{n} \tau}} \quad \text { and } \quad \gamma=s \sqrt{\frac{\tau}{D_{n}}},
$$

where $e$ is the thickness of the layer, $\tau$ is the free carrier life time, $\alpha$ is the coefficient of absorption, $D_{n}$ is the electron diffusion constant and $s$ is the recombination speed in surface.

For these materials, whose permitted transitions are direct, the relation binding $I_{\mathrm{ph}}$ to the incident energy $h \nu$, deduced from the relation (1) is

$$
\left(I_{\mathrm{ph}} h \nu\right)^{2} \approx B\left(h \nu-E_{\mathrm{g}}\right), \quad B=\text { const. }
$$

Figure 6 shows the almost linear variations of $\left(I_{\mathrm{ph}} h \nu\right)^{2}$ versus the incident energy. While extrapolating these curves to the $x$-axis, we deduced values of $E_{\mathrm{g}}$ 


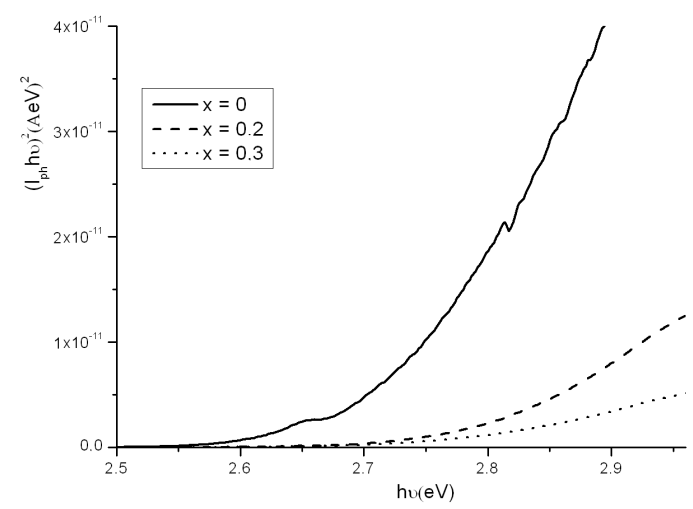

Fig. 6. Variation of $\left(I_{\mathrm{ph}} h \nu\right)^{2}$ as a function of the energy $h \nu$ for different ratio $x$.

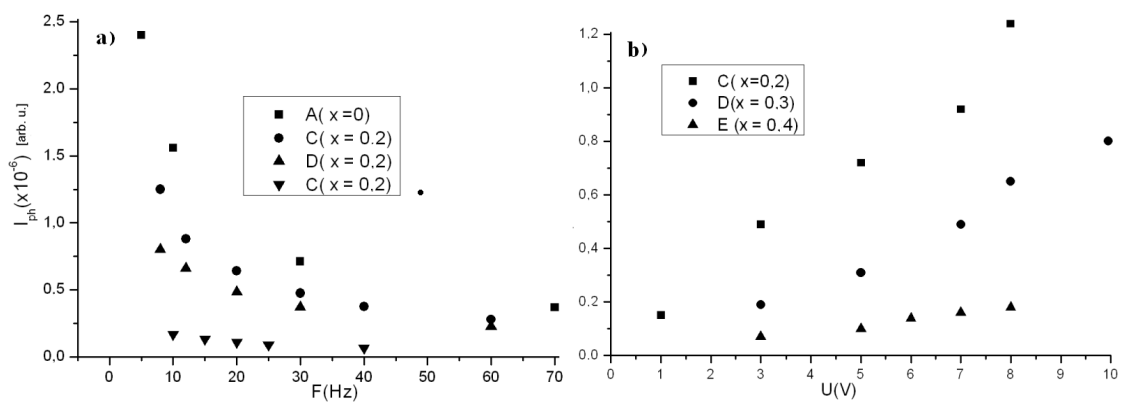

Fig. 7. (a) Photoconductivity measurements of $\mathrm{In}-\mathrm{Zn}-\mathrm{S}$ thin films as a function of frequency modulation as bias voltage $V=10 \mathrm{~V}$. (b) Photoconductivity measurements of In-Zn-S thin films as a function of bias voltage $(f=8 \mathrm{~Hz})$.

that vary from 2.68 to $2.9 \mathrm{eV}$. These values are near enough of those gotten from the reflection and transmission optical measurements.

In the same way, we exploited measures of photoconductivity $I_{\mathrm{ph}}(\lambda)$ done to different polarization voltages and different frequencies, to follow the evolution of $I_{\mathrm{ph}}(V)$ and $I_{\mathrm{ph}}(f)$, Fig. 7. We note a progressive decrease in the intensity of the signal with $x$. These observations are probably explained by the presence of the structural defects and presence of secondary phases as zinc sulphides whose presence becomes more obvious particularly in samples of strong zinc doping $(x=0.3)$. The presence of these secondary phases limits layers photoconductive properties. This phenomenon is observed for all ranges of polarization voltage as well as for all the range of frequencies.

Besides, the reduction of the signal can also be assigned to impurities and defects that trap the photocarriers generated in surface and in depth. The photoconductivity of these thin layers being bound directly to the mechanism of photocarriers recombination, the presence of impurities in layers of high composition can explain this decrease in the photocurrent. 


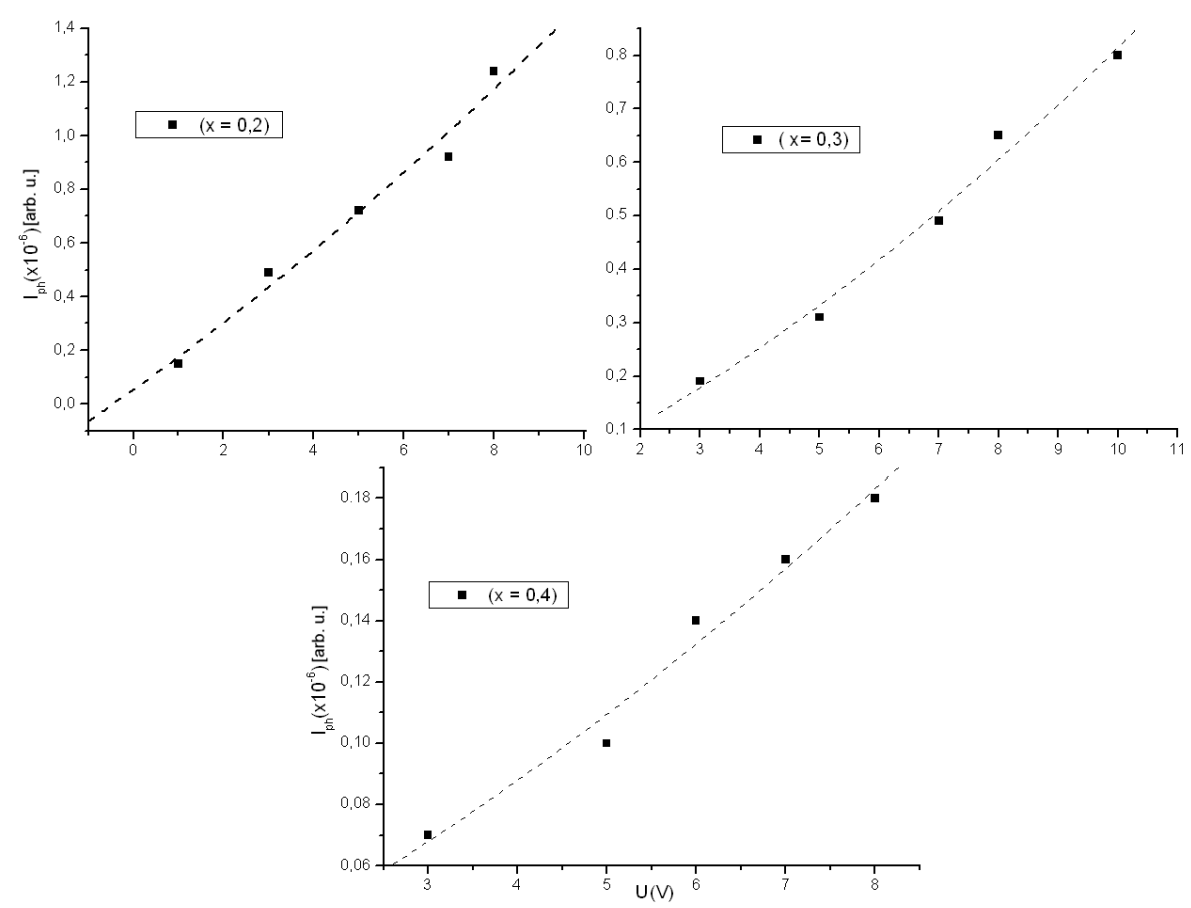

Fig. 8. Photoconductivity measurements of In-Zn-S thin films $(x=0.2,0.3,0.4)$ as a function of bias voltage $(f=8 \mathrm{~Hz})$.

On the other hand, the variation of $I_{\mathrm{ph}}$ according to the polarization voltage follows an empiric law of the shape: $I_{\mathrm{ph}}=a+b V+c V^{2}$, Fig. 8. The linear part is controlled by the $\mathrm{Ohm}$ law that generally exists for the weak polarization voltages. For the elevated voltages, the evolution is rather parabolic obedient to the Child law [12]:

$$
I_{\mathrm{ph}}=\frac{8 \varepsilon \mu_{n} \theta}{9 D^{2}} V^{2},
$$

where $\varepsilon$ is the dielectric constant of the material, $\theta$ is the constant free-to-trapped carriers ratio, $D$ is the mean size of grains and $\mu_{n}$ the mobility of carriers. In this last case the phenomenon of phototransport is limited then by the space electrical charge. Dielectric constant values $\varepsilon=\varepsilon_{0}\left(n^{2}-k^{2}\right)$ of these alloys have been estimated from values of the refraction index $n$ and the extinction coefficient $k$. These last parameters are obtained by the analysis of the optical transmission and reflection spectra by using the iterative and non-recurrent Muller method [13].

The effect of the zinc introduction in these layers is also explained by the reduction of the term $\mu_{n} \tau_{n}$ (Table IV) in accordance with the relation [14]:

$$
I_{\mathrm{ph}}=I_{\mathrm{ph} 0} \frac{\mu_{n} \tau}{l^{2}} V
$$

This relation is valid when the thickness of the $e$ sample is very superior to the 
diffusion length $L(e \gg L) . I_{\mathrm{ph} 0}$ is the total current generated by the incidental photons, $\tau$ is the free carrier life span and $l$ is inter-electrodes distance.

TABLE IV

Optical and electrical parameters of In-Zn-S thin films with the zinc content.

\begin{tabular}{c|c|c|c|c|c|c}
\hline \hline $\begin{array}{c}\text { Composition } \\
x\end{array}$ & $\begin{array}{c}D \\
{[\mathrm{~nm}]}\end{array}$ & $n$ & $k$ & $\begin{array}{c}\varepsilon\left(10^{-12}\right) \\
{\left[\mathrm{F} \mathrm{m}^{-1}\right]}\end{array}$ & $\begin{array}{c}\mu_{n} \theta \\
{\left[\mathrm{cm}^{2} /(\mathrm{V} \mathrm{s})\right]}\end{array}$ & $\begin{array}{c}\mu_{n} \tau \\
{\left[\mathrm{cm}^{2} \mathrm{~V}^{-1}\right]}\end{array}$ \\
\hline 0 & 40 & $2.395^{a}$ & $0.12^{a}$ & $50.64^{a}$ & $11.85 \times 10^{-17}$ & $10^{-6}$ \\
0.2 & 30 & $2.06^{b}$ & $0.12^{b}$ & $37.42^{b}$ & $2.03 \times 10^{-17}$ & $0.6 \times 10^{-6}$ \\
0.3 & 36 & $2.10^{b}$ & $0.10^{b}$ & $38.93^{b}$ & $3.78 \times 10^{-17}$ & $0.3 \times 10^{-6}$ \\
0.4 & 42 & $2.09^{b}$ & $0.17^{b}$ & $38.40^{b}$ & $1.62 \times 10^{-17}$ & $0.08 \times 10^{-6}$ \\
\hline
\end{tabular}

${ }^{a} \lambda=470 \mathrm{~nm} ;{ }^{b} \lambda=415 \mathrm{~nm}$

It is obvious that the optical parameters $(n, k, \varepsilon)$ remain relatively insensible to the introduction of zinc in the sulphide zinc material. Oppositely, when $x$ increases and with the exception of the $\mu_{n} \theta$ parameter $\mu_{n} \tau_{n}$ term decreases in particular for the In-Zn-S prepared with $x=0.4$. This deterioration can be explained by the non-negligible presence of the secondary phase $\mathrm{ZnS}$ in the ternary $\mathrm{ZnIn}_{2} \mathrm{~S}_{4}$. In fact, the presence of this phase influences considerably on the life time of the carriers.

Besides, these results show that when $x$ increases, traps increase and accentuate the phenomenon of carrier recombination at the level of grain boundaries and therefore alter the photocurrent response. The same phenomenon has been noted before on thin layers alloys of $\mathrm{In}_{2-2 x} \mathrm{Al}_{2 x} \mathrm{~S}_{3-3 y} \mathrm{O}_{3 y}$ type [11].

\section{Conclusion}

In-Zn-S semiconductors thin layers have been prepared by the spray technique. This paper shows that both the structural and optical properties of these layers depend mainly on the zinc-to-indium ratio. In the same way, for a composition $x=0.4$, the synthesized layer is quite entirely composed of $\mathrm{ZnIn}_{2} \mathrm{~S}_{4}$ ternary material. On the other hand, the photoconductivity investigation shows that the introduction of zinc alters the carriers conduction mechanism. This result can be considered as very encouraging since a costless and simple spray pyrolysis technique has been used to prepare $\mathrm{ZnIn}_{2} \mathrm{~S}_{4}$ material.

Actually, the electrical study of these layers is in progress (conductivity, Hall effect). In the same line, we intend to test the $\mathrm{ZnIn}_{2} \mathrm{~S}_{4}$ ternary compound as an optical window with absorber ternary layers like $\mathrm{CuInS}_{2}$ and $\mathrm{Cu}_{3} \mathrm{SnS}_{4}$ [15] in order to study the conversion solar energy of $\mathrm{ZnIn}_{2} \mathrm{~S}_{4} / \mathrm{CuInS} \mathrm{S}_{2}$ and $\mathrm{ZnIn}_{2} \mathrm{~S}_{4} / \mathrm{Cu}_{3} \mathrm{SnS}_{4}$ photovoltaic cells. 


\section{References}

[1] T.T. John, M. Mathew, C.S. Kartha, K.P. Vijayakumar, T. Abe, Y. Kashiwaba, Solar Energy Mater. Solar Cells 89, 27 (2005).

[2] L. Bhira, T. Ben Nasrallah, J.C. Bernède, S. Belgacem, Mater. Chem. Phys. 72, 320 (2001).

[3] O. Vigil, O. Calzadilia, D. Seuret, J. Vidal, Solar Energy Mater. 10, 139 (1984).

[4] S.A. Lopez-Rivera, A.J. Mora, D. Acosta Najorro, A.V. Rivera, R. Avila Godoy, Semicond. Sci. Technol. 16, 367 (2001).

[5] E.J. Johnson, Semicond. Semimetals 3, 153 (1967).

[6] A. Serpi, J. Phys. D, Appl. Phys. 9, 1881 (1976).

[7] A. Bosacchi, B. Bosacchi, S. Franchi, L. Hernandez, Solid State Commun. 13, 1805 (1973).

[8] M. Guzzi, E. Grilli, Mater. Chem. Phys. (Elsevier) 11, 295 (1984).

[9] S.I. Radautsan, V.F. Zhitar, V.G. Railyan, Sov. Phys. Semicond. 9, 1476 (1976).

[10] L. Bhira, H. Essaidi, S. Belgacem, G. Couturier, J. Salerdenne, N. Barreau, J.C. Bernède, Phys. Status Solidi A 181, 427 (2000).

[11] L. Bhira, S. Belgacem, J.C. Bernède, J. Appl. Phys. 92, 5327 (2002).

[12] N.F. Mott, R.W. Gurney, Electronics Process in Ionic Crystals, New York 1940.

[13] S. Belgacem, R. Bennaceur, Rev. Phys. Appl. 25, 1245 (1990).

[14] H. Mathieu, Physique des semiconducteurs et des composants eléctroniques, Ed. Masson, Paris 1996, p. 463.

[15] M. Bouaziz, J. Ourfelli, M. Amlouk, S. Belgacem, Phys. Status Solidi A 204, 3354 (2007). 